\title{
Chapter 6 \\ The Role of Context for Crisis Management Cycle
}

\author{
Florence Aligne and Juliette Mattioli
}

Thales Research and Technology, France

\begin{abstract}
This chapter establishes the major role of the sense making and situation-understanding process in crisis management, and outlines the importance of the contextualisation of information in this process. As a result of a wider analysis of past crisis-management feedback, we define the term crisis and propose a crisis-management cycle, along with a set of decision support activities. From a system point of view, crisis management functionalities are structured along three crucial steps: information gathering, situation understanding, and decision making. For each step, the processes involved are described and for each one some relevant techniques are proposed to implement the processes. For the information-gathering step, the use of ontology allows the building and structuring of a coherent situation model. The initial overall picture of the situation, obtained by some on-line information extraction and fusion, is then consolidated in the situation understanding step to provide meaningful real-time situation awareness. This provides the essential base to derive the final decision-making step. In the decision phase, the context has a dual impact on the decision-making process; the context first constrains the resolution of the resource allocation problem, but it also contributes to discriminate between several resource allocation solutions. It is thus shown that each step of the crisis management process relies on the availability and quality of the crisis context, and that this in-time contextualisation is required to enhance the overall process of crisis management.

To summarise, this chapter highlights the key role of situation understanding for crisis management and reveals the crucial necessity of in-time contextualisation at each step of the crisis management process.
\end{abstract}

Keywords Information contextualisation - Situation understanding - Decision support • Crisis management • Ontology • Information fusion $\bullet$ Complex event processing

\footnotetext{
F. Aligne $(\bowtie)$

Thales Research and Technology, France

e-mail: florence.aligne@thalesgroup.com
} 


\section{Introduction}

It is asserted that in the future the number, intensity, and diversity of crises will increase (due, for example, to new asymmetric threats, environmental changes, globalisation, and migrations), and that there is a keen necessity to provide crisis managers with efficient decision support. This need has been identified by the European States, and is present in the security theme of the European Research Framework Programme (FP7 security call 2).

Anticipating, responding, or recovering from a disaster, an incident, a crisis, or an emergency (called later in the chapter as DICE) in a timely and effective manner can reduce deaths and injuries, contain secondary effects, and mitigate the resulting economic losses and social disruption. Implementation of those activities involves information management, decision making, problem solving, tasking and planning, resource management, monitoring, and coordination. These activities are complex multidimensional processes involving a large number of inter-operating entities (teams of humans and systems) and are affected by various social, medical, geographical, psychological, political, and technological factors. From the information technology viewpoint, those processes can be hindered by a lack of adequate, comprehensive and timely information; the presence of conflicting goals, policies, and priorities; or a lack of effective coordination between different rescue operations. Therefore, the situation understanding issue is one of the cornerstones of the DICE management process. To underline that purpose, consider that during the first hours post-incident, the Crisis Response Coordination Team (CRCT) is confronted with uncertainties in making critical decisions. There is a real need to gather, in-time, situational information (e.g., information on casualties), together with contextual information (e.g., medical facilities, rescue and law enforcement units). Then, the sense-making process allows the CRCT team to develop a picture of the overall impact of a DICE and establish priorities for response and recovery efforts. Moreover, the analysis of past crises, such as the Madrid train bombings, Gustav hurricane, or the poliomyelitis propagation, illustrates the need for in-time situation understanding in crisis management based on situational and contextual information.

This need to get a global and contextual situation understanding to mitigate DICE impacts has been illuminated in a study carried out by Thales Research \& Technology, aimed at identifying adapted decision support tools to facilitate the decision-making of crisis managers. This situation understanding is required through the crisis cycle; thus, a main decision-support goal is to provide real-time contextualised information. Real-time in this chapter refers to the notion of on time. It could be only a few seconds or several hours or even some days, depending on the DICE type. For example, in case of a bomb explosion, every second is important to assess the damage and send rescue operations to save lives. Alternatively, in case of a pandemic, the situation evolution is more on a day timescale.

This chapter thus highlights the role of sense making and situation understanding to enhance crisis management decision support. More specifically, it reveals the 
need of real-time context availability in the crisis management process. The chapter is structured as follows: Sect. 2 illustrates the need for timely context in crisis management using the analysis of past crisis feedback. In Sect. 3, as a pre-requisite, the definition of the crisis concept is proposed, together with the key functions required for a crisis-management decision-support system. Section 4 presents the overall process of DICE management through its three phases - information gathering, situation analysis, and decision making - and highlights the need to get contextual information for each phase. Finally, it states that in-time contextual information is also required in the overall upgrade process of DICE management, which includes feedback analysis and training. The conclusion is presented in Sect. 5.

\section{Need of Context for Crisis Management}

Efficient DICE management requires a good, accurate understanding of the situation to improve chances of a successful outcome. Significant challenges include coordination of information amongst entities involved, needs, sharing situation awareness amongst all relevant units, and having an efficient adaptive organisational structure that can change to suit the needs presented by the dynamic situation. This imposes very accurate knowledge of the situation and a keen necessity to correlate the events amongst each other: that is, to get some contextual information. This correlation has to be performed in real time so as to provide an overall and updated understanding of the situation. To illustrate that purpose, here are some real cases.

Poliomyelitis propagation, World, 2003-2005: The international propagation of the poliovirus in 2003-2005 was a shock, as it was considered to be dying out. Due to resurgence of the virus, a wide monitoring infrastructure was created to trace any poliomyelitis cases detected throughout the world. This infrastructure also monitors many other diseases that can be prevented by vaccine. A holistic situation awareness picture is mandatory to watch the world health, to raise the alarm for potential threat, and to anticipate the response. This picture is not only based on situational information (e.g., diseases information) but also on contextual information (e.g., available infrastructures and drugs).

Heat wave, France 2003: As a result of one of the most severe heat waves, more than 14,000 people died in France, mostly elderly people in the northern part of the country where people were not used to the heat. One of the main problems was locating the old people who needed assistance. While trying to minimise the crisis, the French administration was blamed for a lack of reactivity and for ignoring warnings from health and emergency professionals. This heat wave, together with an unusual increase of the paediatric epidemic diseases in winter 2003-2004, outlined the necessity to reinforce the sanitary watch and get some tools to monitor and control hospital emergency services. These events led to the creation of a new regional centre to monitor and drive emergency services, the Centre Régional de Veille et d'Action sur les Urgences (CERVEAU) in the Île-de-France region (CERVEAU 2006; Lepée and Camphin 2007). The two main missions of CERVEAU are to insure: 
- sanitary monitoring of emergency services (situational information), and

- monitoring of care resources (contextual information, e.g., number of available hospital beds...).

These missions confirm the necessity of getting a real-time global context of the sanitary situation to handle the crisis response.

Madrid train bombings, Spain, 2004: A series of coordinated bombings in four Madrid commuter trains occurred during rush hour on the morning of Thursday, 11 March 2004. Soon after, the communication network shut down. Thanks to the knowledge of the context, the emergency units could build a first raw estimation of the damage and severity level. In this case, the context enabled them to provide an assessment of needs despite the lack of communication.

As for organisation of the dispatch of victims to the hospitals, instead of going to the Fire-Emergency Medical Services (EMS) triage centre, about $80 \%$ of the casualties by-passed it and went directly to the closest hospital. This self-evacuation resulted in an over-triage rate of $68 \%$, with the closest hospitals being over-run. In case of a nuclear or biological attack, these hospitals could have been contaminated (PeralGutierrez de Ceballos, Turégano-Fuentes, Pérez Díaz et al. 2005; Turégano-Fuentes, Pérez-Díaz, Sanz-Sánchez, and Ortiz Alonso 2008). Here, lack of a real-time overall view and context of the situation impeded the coordination of the rescuers, prevented the optimal crisis response, and could have increased the mortality.

Hurricane Gustav, New Orleans, USA, 2008: Gustav, the second major hurricane of the 2008 Atlantic hurricane season, caused serious damage in Haiti, the Dominican Republic, Jamaica, the Cayman Islands, Cuba, and the USA. In the USA, there were 138 deaths attributed to Gustav. Due to the anticipation of meteorological conditions, two million people were evacuated to mitigate the risk. Therefore, in the case of the USA, accurate knowledge of the context and anticipation of the threat enabled the evacuation of people and saved lives.

Aftermath of the terrorist attack, USA, 11 September 2001: The 2001 terrorist attacks illustrate the difficulties of governments to tackle a new threat and adapt their defence to a new form of terrorism (isolated cells no longer linked to any state). For J.-L. Bruguière, a French judge specialising in antiterrorism, governments have to face a diffluent and mutant system, which forces one to have a clear and holistic understanding of the situation and the ability to isolate weak signals from amongst a huge amount of information. He insists that it is compulsory to have a geopolitical watch, which implies having a good knowledge of the context (Bruguière 2008). As, by definition here, a "weak" signal refers to some events that, taken independently, would not allow the identification of a risk, but that, when the context is taken into account, are able to reveal some threat through their relations to other events.

Road traffic jam, France, 1999: It started to snow in the late afternoon over the Île-de-France region, when rush hours commenced. Due to a lack of anticipation and the heavy traffic load, it was impossible to clear the road network. Several million people remained blocked until well into the night. To avoid such situations, seven regional centres are now in charge of road traffic monitoring and coordination. 
Their mission is to provide traffic and road information to the users, inform and advise authorities in charge of crisis management, and coordinate emergency traffic response. As for the coordination, it is essential to anticipate the consequences of any actions on the road traffic load (e.g., it is essential to anticipate lane-closing consequences). Also, these centres today benefit from a large amount of traffic data reporting, although according to an operator, they still lack some efficient tools to compare relief roads and estimate the impact of DICE on the traffic.

These examples reveal the critical necessity to get the right information in the right place at the right time. It confirms that the lack of the relevant information in time can have tremendous impact, and that even for events of a long duration, such as a pandemic, the mitigation of DICE relies on the availability and timeliness of an overall and contextualised understanding of the situation.

This, thus, underlines the major role of sense making to support overall crisis management, and shows that real-time information contextualisation is a cornerstone in each phase of the global process from the early watch to the decision.

The next section defines the crisis and crisis management concepts and outlines the main crisis management support functionalities. This provides a framework to analyse the role of context for crisis management (Sect. 4).

\section{Crisis Management}

A research study, carried out in Thales between 2007 and 2008, investigated decision tools for crisis management support (Gabaix and Gavard 2008). Indeed, even if there are some experts specialised in crisis management, there exist few studies that put the crisis systems in the centre of the analysis (systems such as those used in crisis response units). Users' needs are still partially stated and specified; the users rely on basic tools to manage the crises and are not aware of the potential provided by the new information technologies. Thus, there is a necessity to tackle the subject with a wider open-mindedness in order to multiply the usages and the concepts. To achieve this goal, the Thales study has been carried out upon many past crises analysis and through the interviews of about 15 crisis managers from the following organisations: RATP - France Parisian underground, SNCF - French National Railway Company, DDSC - French Direction of the Civil Defence and Security. The main results are presented hereafter.

\subsection{Crisis Characteristics}

Dealing with decision tools for crisis management support immediately raises the question of crisis definition and characteristics, which in turn raises some paradoxes. 
To define the crisis concept, we retain - from the etymology analysis - the notions of bifurcation, lack of time, the decisive nature, the stakes, the breakdown aspect, and the alert signals, as associated with the crisis.

Nevertheless, the analysis of feedback from past crises experience shows that crises differ by their duration, their spatial extent, and their type (terrorist attack, natural disaster...). The multiplicity, the specificity, and the variety of past crises are large; so the Thales approach is not meant to be exhaustive, and instead, prefers to grab some pinches of the crisis characteristics in several domains. (This method is referred to by Rosier as an approach by "touches" (Rosier 2007).)

To get an indication of the variability of past crises, the study covered several types of crises, such as terrorist attacks (like the events of 11 September 2001 in the USA, the bomb attacks of 11 March 2004 in Madrid, the sarin attack in Tokyo subway on 20 March 1995, and attacks in a Paris subway in 1995); natural disasters (with the analysis of the tsunami in Asia in 2004 and hurricane Katrina in Louisiana in 2005 (Lagadec 2007)); industrial accidents (with the explosion of AZF factory in Toulouse (France) in 2001, the electrical blackout in the Northeast of the USA and Canada in 2003, and the power cut in Italy in 2003); and sanitary crises (with the study of SARS in 2003, the mad cow disease (BSE) in 1996, the heat wave in France in 2003, the epidemic of chikungunya at Reunion Island in 2005-2006 (Ministère de la Santé et des Solidarités 2007)). Other types of crises were also considered in the study, together with situations with high potential to turn into crisis ("crisogen" situations), in particular social situations, through the analysis of big gatherings (for example football meetings, demonstrations), of drug trafficking and of independent armed groups (such as the increase of forces of opposition groups as the FARC in Colombia), the cybernetic attacks (Estonia 2004), and the attacks on banking systems.

So, using the feedback on these past experiences, we have retained the criteria proposed by Doctor General Crocq to characterise crises (Crocq 2007):

- A break (disorder, disturbance),

- A bifurcation (transition period, decisive moment, choice),

- A threat (danger, severity),

- An important stake (large number, large scale),

- The urgency (surprise, miss time),

- A degraded, unordered situation (means shortage), and

- A difficulty, a psychological tension.

The validation of one or more of these criteria is then a sufficient condition to qualify the event as a crisis (to a certain level, of course).

Based on the previous analysis, let us retain the following crisis definition (Aligne 2009):

A situation of break [from a normal situation] with regard to the previous events, which threatens the functioning and the values of the individual and/or the community, and where [there] appears an urgent necessity to act despite the degradation of the means, the information and the control. The characteristic of a crisis is that it is unpredictable, out of frame, that it exceeds the existing means, and that it cannot be anticipated by scenarios. 
This definition identifies the crisis as an exceptional situation because of its unpredictable character and the underlying overtaking of means; and as thus, most of the usual methods of intervention will not be adapted. What is crucial for the resolution of the crisis is the capacity and ability to get some tactical reflection and intelligence that can be mobilised. This need was well understood by Lagadec (Beroux, Guilhou, and Lagadec 2007) with his concept of "strengths of fast reflection" units, set up in EdF (French Electricity board). These structures aim at facilitating work of reflection. This outlines the important and essential role of reflection and intelligence in the resolution of the crises. It reveals the necessity of having some information systems and decision-making supports to assist and facilitate reflection.

Let us now characterise such a system.

\subsection{From Crisis Management Concept to Crisis Management Support}

To approach the notion of crisis management, let us first model the crisis. Based on past crisis feedback analysis, we propose a crisis cycle model (Fig. 1) (Aligne and Mattioli 2008), adapted from the Disaster Risk Management Cycle (DRMC) Diagram proposed by Barnier and Baas et al. (Barnier 2006; Baas, Ramasamy, Depryck, and Battista 2008). This crisis cycle is divided into three phases: Predisaster, Response, and Post-disaster.

In the Pre-disaster phase, we identify the activities of watching, monitoring, intelligence gathering, detection, identification of the crisis, and assessment of induced risks.

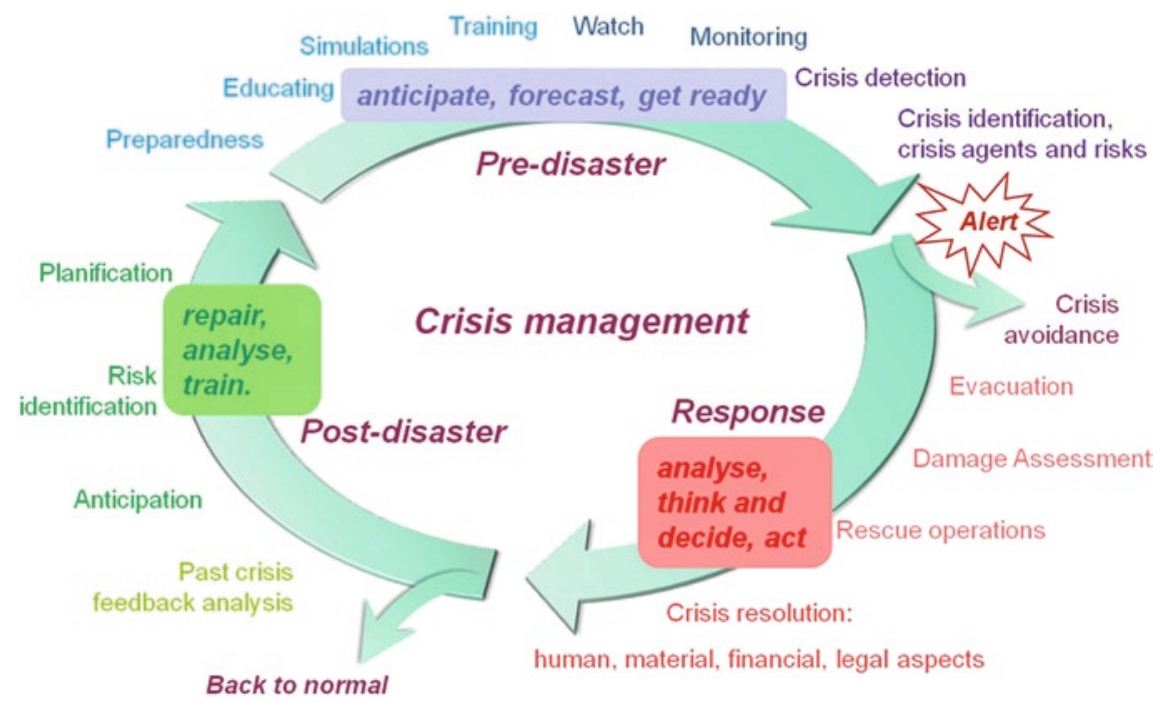

Fig. 1 Crisis management cycle (adapted from Baas et al. 2008 crisis cycle) 
Unless the crisis is avoided, the next phase is the Response phase, which starts with the alert and possibly evacuation procedures. Activities for this phase are evaluation, rescue commitment, and crisis management of different aspects (human, material, financial, and legal). This phase can borrow some methods from defence services, such as the preliminary sorting of the victims to manage a large number of victims. This phase leads back to a normal (stabilised) situation, and is more or less long and expensive depending on the resilience capacity of the organisation. From the beginning of the crisis, experience feedback procedures must be applied to enable an accurate analysis of the crisis in the third phase, the Post-disaster phase.

In the Post-disaster phase, experience feedback is analysed so as to better anticipate and forecast potential problems, to identify the risks and to revise planned procedures. We then enter a process of preparing, teaching, simulating, and training.

It is manifest that this cycle hides some subtleties; for example, the analysis of the experience feedback will start before everything is solved; also, during long term and progressive crises like pandemics or sanitary crises, identification of risks or evaluation of damage must be continuously performed and revised throughout the DICE.

Using this crisis management cycle representation, and based on past crisis analysis (Dautun 2004; Lagadec 1993; Lagadec 2007; Ministère de la Santé et des Solidarités 2007), a set of functionalities for crisis management decision support had been identified. To collect some users' requirements, this work was completed by some operational risk manager interviews in several domains: public transport, hospitals, fire brigades, civil safety administration, and food chain suppliers. The outcome of the study was a set of decision support functionalities that are of particular interest for crisis management, such as information gathering, information updating, information sharing, situation analysis and understanding, situation monitoring, detection and identification of crisis, issuing alerts, decisions set elaboration and presentation, resources allocation, analysis of decision impact (Fig. 2).

These functionalities can be structured into the three main steps of the decision support processing: information gathering, situation analysis and understanding, and decision-making support.

Information gathering: These are the functions allowing the description of the real world in the form of numeric data, which can be worked out by a computer (video, audio, satellites, radars data, etc.), but also of data collected by someone (intelligence gathering). These data have to satisfy certain qualitative criteria so that they can be used. In many domains, the data are still manually entered by an operator into the systems. The functionalities involved are thus information gathering, information updating, and information sharing.

Situation assessment and understanding: These are the functions that enable the processing of data into relevant information to achieve an objective and with regard to the asked question. It includes, for example, functions provided by 


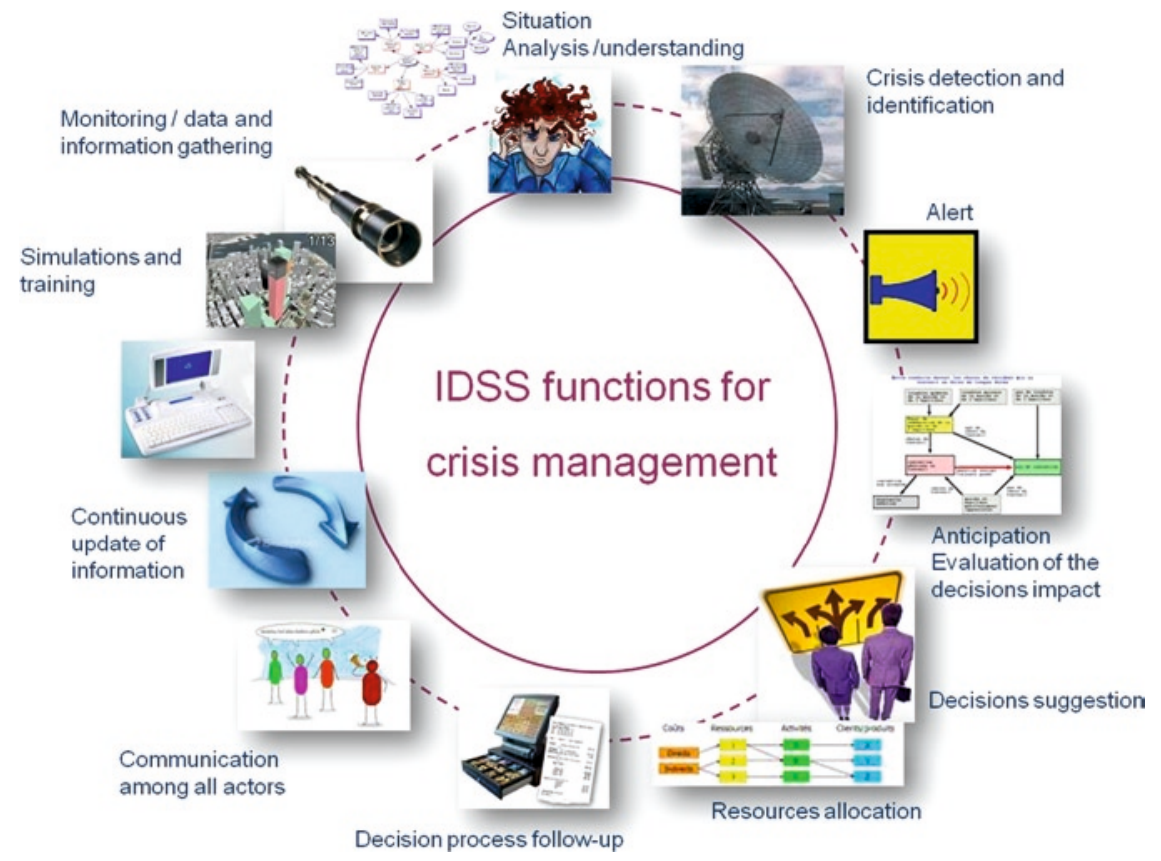

Fig. 2 Crisis management functionalities

geographical information systems (GIS), by the information systems of command centres, biometric systems, and statistical tools of analysis. The assessment and understanding of the situation will provide the user with a clear and synthetic vision of the situation, enable the organisation and analysis of the information, and facilitate the decision-making process. This, thus, comprises the production of key indicators, dashboards, etc. The functionalities involved are situation analysis and understanding, situation monitoring, detecting and identifying the crisis, and issuing alerts.

Decision-making support: These functions assist decision makers to overcome the complexity of the situation by suggesting or supporting decisive alternatives. We can mention, for example, the planning and scheduling systems of enterprise resource management (ERP). This domain still has much to explore. The decision making very frequently relies on the combination of expert assessments and complex logics. Although we do not aim to replace the expert, to support him in his decision task can turn out to be very delicate and complicated. Some systems, used in well-delimited domains, nevertheless have already proven their effectiveness. The functionalities involved are thus the elaboration and presentation of the decision set, resources allocation, and analysis of decision impact.

In the following section, we describe the processes involved in these three steps and propose some relevant techniques for implementation of each one. This will highlight the key role of "context" in crisis management. 


\section{Contextualisation Use in the Three Phases of DICE Management}

\subsection{Contextualisation for Information Gathering}

The specific term "situation awareness", most commonly used by the HumanComputer Interaction community (Endsley 1995), is defined as "the perception of the elements in the environment within a volume of time and space, the comprehension of their meaning, and the projection of their status in the near future". Furthermore, a situation can provide answers to some but not all questions induced by the decision problem. Therefore, situation awareness plays a central role in cognition, which spans the entire spectrum of cognitive activities from situation analysis (perception + comprehension) to near-future forecast. To efficiently support humans in their situation understanding in such decision-making processes and particularly in the induced cognitive overload context, there is a crucial need to provide in time only the critical information required. In such situation, the decision makers have to deal with a huge amount of heterogeneous information that comes from different sources and thus is often redundant, if not conflicting.

It is therefore necessary to model and specify the context and situation to provide support functions for cognitive overload mitigation such that the stakeholders can easily exchange, share, and re-use their knowledge. Situation understanding is not only a matter of knowing the objects that are present in the external world, but also to make sense of the situation assessment achieved by specifying and representing the relations amongst it. To design such support systems, one of the major challenges is to construct a situation model from which crisis management operations can be planned and updated. A situation model is a representation of the situation that will serve as a reference for each participating user; it allows a complete cognitive understanding of the situation (Mattioli, Museux, Hemaissia, and Laudy 2007). It includes the representation of all events, actions, actors, past experiences, and items which explain, impact or are impacted by the situation (note that in this chapter, actors are the individuals involved in the DICE mitigation). Therefore, the situation model provides a cognitive representation of the situation context.

To build a situation model, we use ontology concepts as they are used to capture knowledge about some domain of interest. Matheus, Baclawski, and Kokar (2003a) advocate the use of formal ontologies to describe the fundamental events, objects, and relations of a situation's domain and logical rules. This is in order to define ways of fusing information and identifying higher order relations relevant to the situation (Matheus, Baclawski, and Kolar 2003b). Smirnov, Pashkin, Shirov, and Levashova (2007) also propose to include ontology for mega disaster management.

Therefore, the situation model contains a knowledge-level view of DICE using some specific ontology, such as Crisis Response Management (CRM) ontology. The model is created and updated by a constant flow of events and reports collected from the operational space. These events include both human and artificial intelligence. Because of the large amount of raw data being collected, the event stream 
needs to be processed and correlated to produce situational events, i.e., events at the domain level. This reduction and inference step is performed by an informationfusion stage. Thanks to the OASIS consortium, most of the relevant situation items (class, property or individual) can be described through the definition of the Tactical Situation Object (TSO) (OASIS Project 2006), which contains at least the following information.

- Identification information: it shall be identified in an unambiguous way. It shall also describe who the originator of the information is and when the information was created.

- Description of the case: the TSO is one solution to provide to other entities its own view of the case such as the type of the case, its extent, the number of casualties, the consequences on the environment, its criticality, etc.

- Description of the context: the CRCT is interested to know which resources are already used, which resources are available (including the human resources), their availability, their position, and their capabilities.

- Description of the actions: it is also very important to inform the others of the activities which are in progress or which are foreseen, so that the coordination is efficient. Information on the past, future, and ongoing tasks must be available (their status, the teams, and resources that are engaged on a task, their planning, etc.).

Following OASIS guidelines, the Interactive Collaborative Information Systems (ICIS) project (2003) provides crisis response management ontology composed of three modules: situational, contextual, and decisional (Figs. 3, 4 and 5). The situational information provides a view of the crisis case, such as, for example, the type of crisis, its extent, number of casualties, environmental impact, and criticality. The contextual information addresses the status of resources, which includes list of resources, their availability, position, and capabilities. The decisional information concerns the tasks that are ongoing. This comprises their status, the team, and resources involved and their planning (Mattioli et al. 2007).

Based on this situation model, the information extraction consists of the analysis of data coming from different sources so that these data become meaningful. The objective is to extract information from the available data. The techniques we use are text mining, audio mining, and image processing. The notion of text mining has been widely spread by the search engines though these do not allow the performance of sense making on the data. Text mining, as used here, seeks to find relations between blocks of text data contained in a text to provide information. Audio mining allows the extraction of information from an audio stream (speech or noise analysis). In particular, speech analysis is reinforced today by the background sounds analysis that can provide much additional information to optimise the sense-making processing. Information extraction methods, either in text, audio or images, usually use ontology to structure the input data, and supervised classification methods to analyse and extract relations amongst entities. Thus, this is very much correlated with the above modelling phase. The capability of the method to extract relationships amongst entities and model the crisis situation context is a key factor of the information extraction phase. 


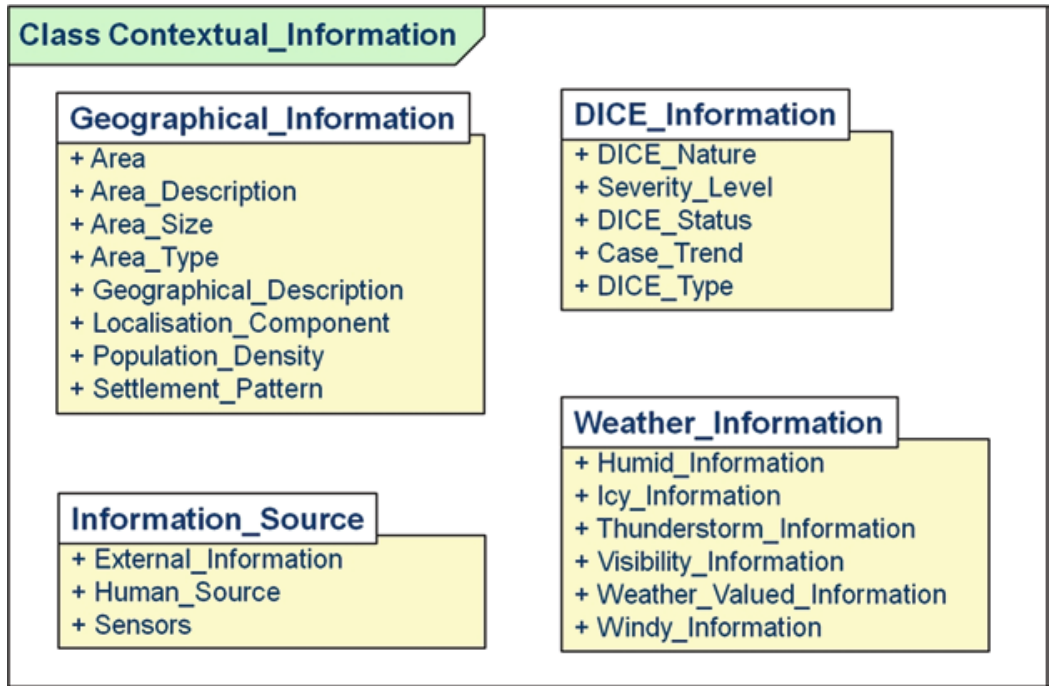

Fig. 3 Contextual information modelling, from ICIS project (ICIS project 2003)

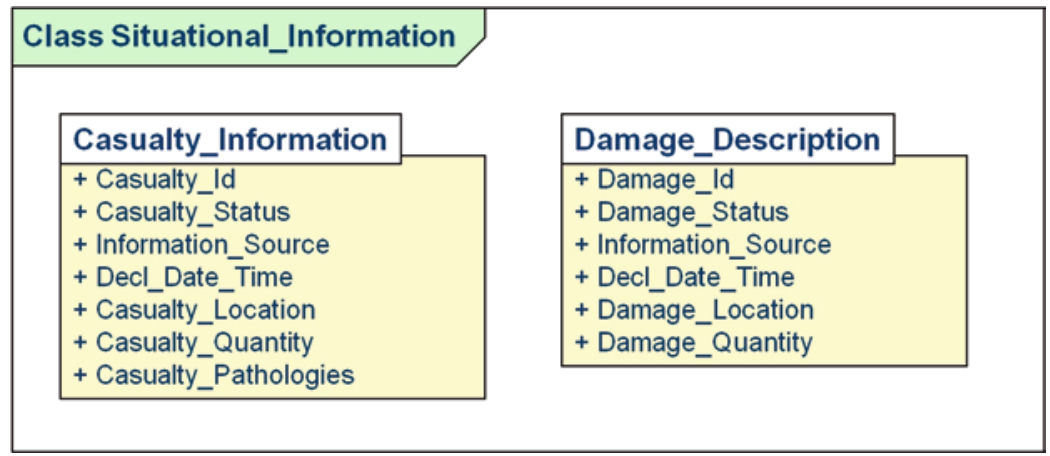

Fig. 4 Situational information modelling, from ICIS project (ICIS project 2003)

Following the information extraction, the construction of a coherent overall situation picture will require some fusion of heterogeneous and redundant information techniques. Most of the time, because information is coming from a wide range of sources including both human and signal sources, the pieces of information must be combined and correlated in accordance with

- The objectives of the crisis response (for example, for early crisis response the objectives can be immediate life saving, search and rescue, medical aid),

- The information reliability and relevance, and

- The information temporality (obsolescence and update).

The processing and correlating of all available information is performed by an information-fusion stage. The information items, stored as graph structures, are combined using fusion heuristics. The fusion algorithm is based on a graph homomorphism 


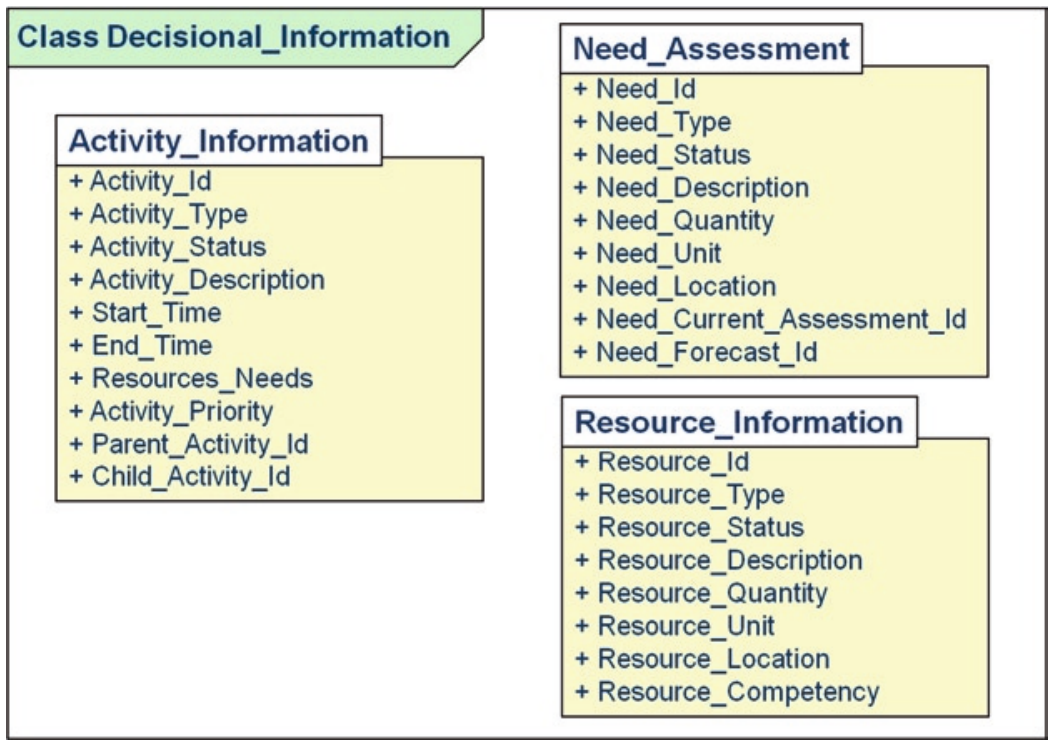

Fig. 5 Decisional information modelling, from ICIS project (ICIS project 2003)

algorithm where we look for compatible subgraphs (i.e., fusible). The compatibility is processed thanks to the fusion heuristics - called fusion strategies - that contain both a part to test the compatibility of two observations and a part to compute the fused observation (Laudy and Ganascia 2008a,b; Laudy, Ganascia, and Sedogbo 2007).

This early overall situation picture then has to be consolidated with some situation understanding process and sense-making analysis. This is to provide a meaningful situation awareness, the basis on which the final decision-making step can be performed. These two steps are presented hereafter.

\subsection{Contextualisation to Get a Meaningful Situation Awareness}

Situation understanding is used to manage, in real time, crisis situations of critical infrastructures (such as production line breakdown, network intrusion or attack of public area). For example, an alert can be raised only if responsible actors have a clear understanding of the global situation. The situation understanding has to be as complete as possible and has to consider the information from different points of view, from the smallest standpoints to wider perspectives. For example, the detection of weak signals amongst a huge amount of information is of major importance for information intelligence activities. This implies a capability of analysing the situation down to its smallest component using a well-established global situation analysis. This should enable the detection of small pieces of information that - put back into the context - are of prior interest. 
The situation understanding process is a cognitive and analytical process by which we can take some information and synthesise it with previously held information. It is then used in a new context, or used in a different way to that envisioned when first collected. The difference between situation understanding and information gathering is the difference between learning and memorising as compared to this ability to transform information through experience and know-how. Thus, the situation understanding depends not only on the information content but also on its contextualisation.

It should be pointed out that context also impacts the frequency at which information used to get meaningful situation awareness is constantly updated. As already mentioned in the introduction, real-time here refers to the notion of on time and can range from a few seconds to many days depending on the DICE type (example of bomb explosion vs. pandemic situations). The need for real-time information for situation awareness is indeed very dependent on the context, as shown in the following example which is very illustrative, though not directly linked to DICE management.

When enhancing air traffic management (ATM) procedures and tools, new concepts have to be validated through real-time simulation exercises. During an air traffic simulation session, pilots were asked to deal with a new ATM procedure that involved an automatic adaptation of the aircraft speed with regard to the preceding aircraft speed and position. The modified simulator displayed the value of the aircraft speed taking into account the new requirements in real time. However, after a few training exercises, this speed value displayed in real time had to be averaged and smoothed so as to display a stabilised value of the speed. This was because pilots were unable to get a comprehensible situation awareness from a constantly changing speed. It is therefore important to adapt the notion of real time to the use, context, and decision need. Thus, real-time here implies the notion of on time, or as referred to (Nelson 2004) time to decision.

This outlines the crucial role of contextualisation in the previous informationgathering step to building a contextualised situation understanding, and emphasises that this information gathering requires real-time updates. Complex event processing (CEP) is widely used for situation analysis and understanding. It allows the identification of relevant information to provide answers, in real time, to the crisis managers' needs. It consists of the detection of complex events, the correlation, and analysis of hierarchical events and events relations (such as causality or temporality) (Museux, Mattioli, Laudy, and Soubaras 2006).

With a keen, updated, and contextualised situation awareness, a coherent and efficient crisis management plan to mitigate and resolve the DICE can be envisaged and elaborated.

\subsection{Dual Impact of the Context on Decision Making}

Decision-making support is used in the response planning and implementation phase to optimise resource allocation and scheduling. This aims to first meet 
specific relief objectives and later to fulfil recovery and development goals. Because of the high combinatory complexity of resource allocation and scheduling problems, we use a hybrid method, combining an optimisation algorithm with a meta-heuristic algorithm. This enables us to split the problem into subproblems. To be efficient and operational, the selected decision has to correctly tackle the operational constraints in the optimisation problem. These constraints then are to be included into the Situation Model. The final decision also takes into account some potential conflicting goals. This last issue can be addressed by the use of some negotiations in the optimisation algorithm (Hemaissia et al. 2006).

Crisis response requires the management of all relevant resources (infrastructures, material, human); a lack of resources can lead to the abortion of the whole response. It is thus very important to have an updated knowledge of the availability of resources and of the procedures required to use them. Besides, in order to support the decision makers, the shared situation awareness picture has to materialise the "decisional environment" and so makes it clear why emergency operation planning is necessary.

Once some allocation or scheduling of resources has been performed, and before the commitment to the crisis response tasks has been made, there is a great necessity to analyse what would be the impact of the response operations. This impact should be analysed in terms of results as well as risk involving the crisis mitigation. Between two responses, it might be wiser not to choose the more efficient one (efficient with regard to some predefined criteria such as time and/or cost) if its level of risk is very high compared to the other one. The efficiency and risk can be evaluated by the modelling of the situation evolution. The final response selection thus requires a time projection of the situation understanding process. We call it the "dual impact of the context for the crisis management decision support". The context first constrains the resolution of the resource allocation problem (or scheduling), but it also helps discriminate amongst several resource allocation solutions.

To measure the impact of a decision to the context, some simulation tools to "play" the decision in advance are used to analyse its consequences. As an example, some recent research carried out by the multiagent community is now presented.

This research aims to define a simulation tool for road traffic crisis management and uses the context as a key function for the agent to take his/her decisions. Environment as active support of interaction (EASI) is a multiagent model of interaction conceived by the Laboratoire d'Analyse et de Modélisation des Systèmes pour l'Aide à la Décision (LAMSADE), which is based on a reification of implicit and explicit rules to enable the interaction of a multiagent system with the environment. The objective is to model the link between the perception domain and the activities of the agent, and so to allow the use by the agent of this knowledge to reach its objective. More recently, a new contextual simulation multiagent model has been studied by F. Balbo and F. Badeig. The objective is to extend the EASI contextual principle of interaction to a contextual activation of the simulation agents (Saunier, Balbo, and Badeig 2007). This model, called Environment as Active Support of Simulation (EASS), separates the simulation process from the activation process of the agents. The approach used is the study of the context and the interactions between the actors 
(Badeig, Balbo and Pinson 2007). A first application of this work concerns crisis management in the transport domain. The goal is to simulate the complexity of a crisis for better analysis and understanding. The simulation of different scenarios for the same crisis will help the conception of new intervention plans and the validation and adaptation of existing plans (Badeig and Balbo 2008).

The above description of the decision-making process thus shows that the situation understanding process is essential and that a decision has to be correlated to its context.

\subsection{Contextualisation of the DICE Management Upgrade Process}

A very important point to take full advantage of DICE management processes and to improve them is to regularly train all the actors who may have to play a role in the DICE mitigation. It must be stressed that people involved in the process during a DICE management might not be people usually in charge of the process. The training should thus be large enough to familiarise the people with a large event of practices. Training sessions aim at getting the people used to the tools and practices, often through life-sized exercises of simulated DICE events. The goal is to put people in some degraded situations already encountered by others, in order to develop individual best practices. This implies getting the actors accustomed to different contexts (Gauthey 2008).

The upgrade of the DICE management process also relies, of course, on the analysis of the feedback from past events so as to learn and capitalise from past mistakes. As stated by Cheila Duarte-Colardelle, most of the incident report forms do not allow for catching the "why" and "how" of the acts and decisions. The situation perception is ignored, which is a limitation to the understanding of some events. Dysfunction and misleads are analysed with the aim of understanding not only the "how", but also especially the "why". When someone makes a decision, several choices are available; the decision and the resulting action might not be understood by others. "Why has he done this?" There is always some reason that can be explained by the context (Duarte-Colardelle 2006).

Rescue plans have also been defined step-by-step and adapted with regard to some evolution of the context. These evolutions are detected and analysed to capitalise on and benefit from them. In the work of Duarte-Colardelle (2006), the author explains how to trace and analyse the dynamic progress of each case study. This method, called positive feedback (REX positif), aims no longer at learning from accidents or dysfunctions, but at detecting the good practices and at strengthening them; the notion of "key moment associated with a cycle of decision" is the most significant contribution of the method. The comparison between the decisions that make the situation worse, with "good" decisions that could have saved the situation, is the basis of the process. We agree with Sarant that this is an important contribution, provided the decision making contexts are taken into account (Sarant 2003). 


\section{Conclusion}

This chapter has established the major role of sense making and situation understanding process in DICE management and has outlined the importance of real-time information contextualisation in this process.

The efficiency and effectiveness of DICE management responses are deeply correlated with the availability and quality of the relevant contextualised information. The appropriate update of this information is a key issue to enhance the DICE management process. Many examples, taken from past crisis experience feedback, outline the keen necessity to get the right information at the right place and in-time. Thus, in the watch process the need to get a holistic situation awareness based on updated situational and contextual information is illustrated by the poliomyelitis example. The necessity to get a holistic geopolitical watch correlated to the context is also stressed by the antiterrorism judge Bruguière on the analysis of the 11 September event.

To summarise, the aim of this chapter has been to present application domain crisis management and to show the importance of the real-time context in this domain. To introduce the subject, past crisis analysis examples were presented in Sect. 2 to illustrate the need of contextualisation at various stages of the crisis management process. Section 3 presents the meaning of crisis management process and the main functionalities involved along three axes: information gathering, situation understanding, and decision making. Using this structure, an analysis of the role of context in the DICE management process was derived in Sect. 4. This analysis demonstrates that contextual information is required at every stage of the DICE management process. It also shows that nowadays some emergent technologies are able to deal with real-time contextualised information to fulfil the requirements of the identified DICE management support tools functionalities. Thus, for the information-gathering step, the use of ontology allows us to build and structure a coherent situation model that enables DICE managers to build and share a real-time contextualised situation representation. The initial overall picture of the situation, obtained by information extraction and fusion and with real-time updates, must then be consolidated in the situation-understanding step to provide meaningful situation awareness. This provides the essential base to derive the final decisionmaking step. In this last step, we have outlined that the context has a dual impact on the decision-making process: the context constrains the resolution of the resource allocation problem and also helps discriminate amongst several resource allocation solutions.

The variety of actors involved and the unpredictability of the situations are the main specificities of the DICE management application domain. The importance and impact of real-time context throughout the DICE management process have been carefully established here, and emergent technology has been identified for each step of the DICE management process to take the context into account. Yet, more research should be conducted for a deeper insight into the robustness of this contextualisation and insure that the foreseen solutions are coherent across the large variety of 
stakeholders and throughout the DICE management processes. DICE management endeavours to deal with a very large variety of actors (medical units, fire brigades, police forces, but also, electricity, water, road infrastructures services...), and situations (located events or disasters that impact a large geographical area, natural events, human mislead or intend attacks...). This implies the need to take into account a broad set of contexts and to enable adaptation in real time the decision support tools for any new situation. The solutions envisaged here are meant to be as generic as possible; there has been no focus on a specific type of DICE as we tackled the problem from the analysis of DICE characteristics. Yet, the adaptability of the solutions for different contexts that occur randomly should be further investigated. In particular, it is expected that there would be great benefit in considering the outcomes of recent researches on the use of improvisation to provide real-time decision making (Stein 2008).

Currently, in Thales Research and Technology we apply this contextualisation process to improve the DICE operations deployment at the DICE management strategic level in a new study carried out within the Descartes project (project of the French "pôle de compétitivité" Infom@gic). This study addresses the crisis cell at the council level and aims at optimising the resources allocation of the first crisis responders (emergency medical units, fire brigades, and police). Following the recommendations presented here, the context is taken into account through the modelling of rescue plans. Though this still requires more theoretical work in the planning domain, the results are promising and enable us to cross different contexts for different actors, both at different space and time scales.

Lastly, we mention another area of research that should be addressed to enhance the whole process of DICE management: the resolution of conflicting goals. It is indeed another major issue to be resolved when many actors are implicated in the decision process. How could the use of context anticipate and prevent the occurrence of conflicting situations, and could it facilitate their resolution?

\section{References}

Aligne, F., Which information and decision support system for the crisis management? in NATO RTO-MP-IST-086, May 2009, Romania.

Aligne, F. and J. Mattioli, Situation understanding process: The cornerstone for crisis management support, in RTDMC 08 Proceedings, 2008, Paris.

Baas, S, S. Ramasamy, J.D. Depryck and F. Battista, Disaster Risk Management System Analysis. A Guide Book, FAO, Rome, January 2008, URL=ftp://ftp.fao.org/docrep/fao/010/ai504e/ ai504e01.pdf.

Badeig, F. and F. Balbo, La gestion de crise dans les transports, Un simulateur multi-agent centré environnement, in Atelier Systèmes d'Information en Transport, Inforsid 08, 2008.

Badeig, F., F. Balbo and S. Pinson, Contextual activation for agent-based simulation, in Proceedings, 21 st European Conference on Modelling and Simulation, 2007, Prague.

Barnier, M., Pour une force européenne de protection civile: Europe aid, 2006, URL=http://ec. europa.eu/commission_barroso/president/pdf/rapport_barnier_20060508_fr.pdf 
Beroux, P., X. Guilhou and P. Lagadec, Implementing rapid reflection forces, Crisis Response, Vol. 3, No. 2, March 2007, pp. 36-37.

Bruguière, J., Le terrorisme, nouvelle menace, Conference held at Polytechnic, 24 January 2008, France.

CERVEAU, Centre Régional de Veille et d'Action sur les Urgences en Île-de-France, Charte de partenariat, 2006.

Crocq, L., Médecin Général des Armées, Formation et sensibilisation NRBC, Haut Comité Français pour la Défense Civile, 2007.

Dautun, C., Organisation de la gestion de crise « Les secours face à une crise de grande ampleur, Thèse professionnelle, mastère Sécurité Industrielle et Environnement, Ecole des Mines d'Alès et INERIS, France, 2004.

Duarte-Colardelle, C., Analyse de la dynamique organisationnelle en temps de crise, Thèse de doctorat en Ingénierie et Gestion - Sciences de Gestion, Ecole Nationale Supérieure des Mines de Paris, France, 2006.

Endsley, M., Toward a theory of situation awareness in dynamic systems, Human Factors, Vol. 1, No. 37, 1995, pp. 32-64.

Gabaix, J.-C. and C. Gavard, Exploration d'un domaine d'innovation dans un marché émergent: systèmes d'information et d'aide à la décision pour la gestion de crise, Master Projet Innovation Conception de l'Ecole Polytechnique, Mention Economie et Gestion, Polytechnique, France, 2008.

Gauthey, O., Le retour d'expérience, Etat des pratiques industrielles, Les cahiers de la sécurité industrielle, ICSI, Institut pour une culture de sécurité industrielle, France, 2008-02.

Hemaissia, M. et al., Cooperation-based multilateral multi-issue negotiation for crisis management, in AAMAS'06 International Workshop on Rational, Robust and Secure Negotiations in Multi-Agent Systems (RRS 2006), May 2006, Hakodate, pp 77-95.

ICIS project, D6 - A survey on situation theory and CEP focusing on emergency situation understanding, ICT project selected for by the BSIK programme, ICIS-ESA cluster, Interactive Collaborative Information Systems, 2003-2008, URL=www.icis.decis.nl.

Lagadec, P., Apprendre à gérer les crises, Editions d'Organisation, 1993.

Lagadec, P., Katrina: Examen des rapports d'enquête, 2007, URL=http://www.patricklagadec. net/fr/pdf/2007-25.pdf

Laudy, C. and J.G. Ganascia, Information fusion in a TV program recommendation system, in Proceedings of the 11th International Conference on Information Fusion (FUSION 2008), July 2008a, Cologne, pp. 1455-1462.

Laudy, C. and J.G. Ganascia, Information fusion using conceptual graphs: A TV programs case study, in additional Proceedings of the 16th International Conference on Conceptual Structures (ICCS 2008), P. Eklund and O. Haemerlé (Eds), July 2008b, Toulouse, pp. $158-165$.

Laudy, C., J.G. Ganascia and C. Sedogbo, High-level fusion based on conceptual graphs, in Proceedings of the 10th International Conference on Information Fusion (FUSION 2007), July 2007, Québec.

Lepée, M. and P. Camphin, Le centre régional de veille et d'action sur les urgences d'île-deFrance (Cerveau): Une information partagée pour une action concertée, Gestion hospitalière, No. 462, 2007, pp. 60-65, France.

Matheus, C., K. Baclawski and M. Kokar, Derivation of ontological relations using formal methods in a situation awareness scenario, in Proceedings of SPIE Conference on Multisensor, Multisource Information Fusion, April 2003a, pp. 298-309.

Matheus, C., K. Baclawski and M. Kolar, Phase I Final Report: A Formal Framework for Situation Awareness, AFRL Funding number: F30602-02-C-0039, January 2003b.

Mattioli, J., N. Museux, M. Hemaissia and C. Laudy, A crisis response situation model, in 10th international Conference on Information Fusion, 2007, Quebec.

Ministère de la Santé et des Solidarités, Chikungunya: gestion d'une crise longue, société française de médecine de catastrophe, rapport, France, 2007. 
Museux, N., J. Mattioli, C. Laudy and H. Soubaras, Complex Event Processing Approach for Strategic Intelligence, FUSION 2006 Technical Program, Paper Number: 200, Tuesday, 11 July 2006, Special Session: Situation Management I.

Nelson, G.B., Real time decision support: Creating a flexible architecture for real time analytics, in SUGI 29 Proceedings, Montréal, May 9-12, 2004.

OASIS Project, Open Advanced System for Disaster and Emergency Management Definition of the OASIS Tactical Situation Object, EU- FP6 - OASIS project - Part of the D-TA2_06 (OASIS external interfaces), 2006. URL=http://www.oasis-fp6.org/documents/OASIS_TA21_ DDD_041_DSF.pdf

Peral-Gutierrez de Ceballos, J., F. Turégano-Fuentes, D. Pérez Díaz et al., Casualties treated at the closest hospital in the Madrid, March 11, terrorist bombings, Critical Care Medicine, Vol. 33, 2005, pp. S107-S112.

Rosier, R., Stratégies et organisations des processus d'exploration: le cas de la pile à combustible chez Axane/Air Liquide, Thèse, Université de Paris-Est Marne-la-Vallée, U.F.R. science et gestion, 2007.

Sarant P.-M., Retour d'expérience sur la prise de décision et le jeu des acteurs: le cas du cyclone Lenny dans les Petites Antilles au regard du passé, Programme 'Evaluation et Prise en compte des Risques naturels et technologiques', Ministère de l'écologie et du développement durable, France, 2003.

Saunier, J., F. Balbo and F. Badeig, Environment as active support of interaction, in Proceedings Environments for Multiagent Systems, LNCS 4389, 2007, pp. 87-105.

Smirnov, A., M. Pashkin, N. Shirov and T. Levashova, Intelligent support of context-based megadisaster management: Hybrid technology and case study, in Proceedings of the 4th International Conference on Intelligent Human-Computer Systems for Crisis Response and Management ISCRAM 2007, 13-16 May 2007, Delft.

Stein, E.W., Supporting real time decision-making: The role of context in decision support on the move, SIG DSS Pre-ICIS Workshop on Real Time Decision Support - RTDMC 2008, 13 December 2008, Paris.

Turégano-Fuentes F., D. Pérez-Díaz, M. Sanz-Sánchez and J. Ortiz Alonso, Overall assessment of the response to terrorist bombings in trains, Madrid, 11 March 2004, European Journal of Trauma and Emergency Surgery, 2008, Æ No. 5, Urban \& Vogel. 